\title{
Emergency management of a patient with severe airway obstruction resulting from poorly differentiated thyroid carcinoma: A case report
}

\author{
RONG YANG ${ }^{1}$, XIONGFEI YU ${ }^{1}$, LIANG MA ${ }^{2}$ and FUSHENG WU ${ }^{1}$ \\ Departments of ${ }^{1}$ Surgical Oncology and ${ }^{2}$ Cardio-Thoracic Surgery, The First Affiliated Hospital, \\ Zhejiang University College of Medicine, Hangzhou, Zhejiang, P.R. China
}

Received April 10, 2012; Accepted July 18, 2012

DOI: $10.3892 / \mathrm{ol} .2012 .827$

\begin{abstract}
We present a case of a life-threatening almost complete airway obstruction resulting from poorly differentiated thyroid carcinoma in a 48-year-old male. Airway obstruction may lead to unexpected mortality by suffocation and patients with poorly differentiated thyroid carcinoma usually have a fast deterioration and fatal outcome. In the case presented, we describe a safe and effective treatment strategy. Assisted by femoro-femoral cardiopulmonary bypass oxygenation, a tracheal stent was implanted successfully. Following surgery there were no complications, and chemoradiotherapy resulted in the relief of obstructing symptoms and improved the quality of life of the patient. This case indicates that femoro-femoral cardiopulmonary bypass provides adequate oxygen support to undergo further management and that tracheal stent implant is an effective emergent measure to relieve severe airway obstruction in patients with poorly differentiated thyroid carcinoma.
\end{abstract}

\section{Introduction}

Poorly differentiated thyroid carcinoma is a relatively rare disease. At present, it is thought to be an intermediate entity in the progression of well-differentiated thyroid carcinoma to anaplastic thyroid carcinoma $(1,2)$. As patients with poorly differentiated thyroid carcinoma usually have a fast deterioration and fatal outcome, this carcinoma presents surgeons with challenging treatment options. Furthermore, when the carcinoma invades the trachea and results in airway obstruc-

Correspondence to: Professor Fusheng Wu, Department of Surgical Oncology, The First Affiliated Hospital, Zhejiang University College of Medicine, 79 Qingchun Road, Hangzhou, Zhejiang 310003, P.R. China

E-mail: wufusheng1962@hotmail.com

Key words: thyroid cancer, airway obstruction, cardiopulmonary bypass, stents tion, the situation is an emergency and may be complicated. Therefore, an appropriate treatment strategy is necessary. In the current study, we present a case of an almost complete airway obstruction resulting from the invasion of poorly differentiated thyroid carcinoma. The anesthetist considered intubation to be dangerous as it had the potential to cause rupture and embolism of the tumor and lead to asphyxia. With the support of cardiopulmonary bypass oxygenation, we successfully implanted a tracheal stent under the guidance of flexible fiber optic bronchoscope. The patient's respiratory function improved and no complications occurred. Furthermore, subsequent concurrent chemoradiotherapy reduced the size of the tumor and prolonged the survival time of the patient.

\section{Case report}

Patient data. A 48-year-old male presented with dyspnea and intermittent stridor for three months. He also complained of a cough and experienced a slight loss of voice. Therefore he attended a local hospital where the physician misdiagnosed the condition as asthma and administered the corresponding treatment. Unfortunately, the dyspnea worsened gradually, and a month later the patient became hoarse. Thus, the patient was referred to our hospital. Informed consent was obtained from the patient prior to the study. The ethics committee of Zhejiang University College of Medicine, Zheijiang, China approved the study.

Palpation of the neck revealed a diffuse and firm goiter without tenderness. Neck and chest computed tomography (CT) scan showed an approximately $6.5-\mathrm{cm}$ goiter in two lateral lobes of the thyroid gland, firmly wrapping the trachea. The goiter had invaded and protruded into the tracheal lumen and the narrowest internal diameter was $3 \mathrm{~mm}$ (Fig. 1). The goiter extended down the trachea into the chest and caused narrowing in a $5-\mathrm{cm}$ stretch of the trachea. Laryngoscopy revealed impaired mobility of the left vocal cords. The results of the thyroid function test were within normal limits. The day after admission, the patient presented with a sudden onset of orthopnea and hemoptysis without an evident cause. After holding group consultations, we selected emergency treatment with the family's consent. 


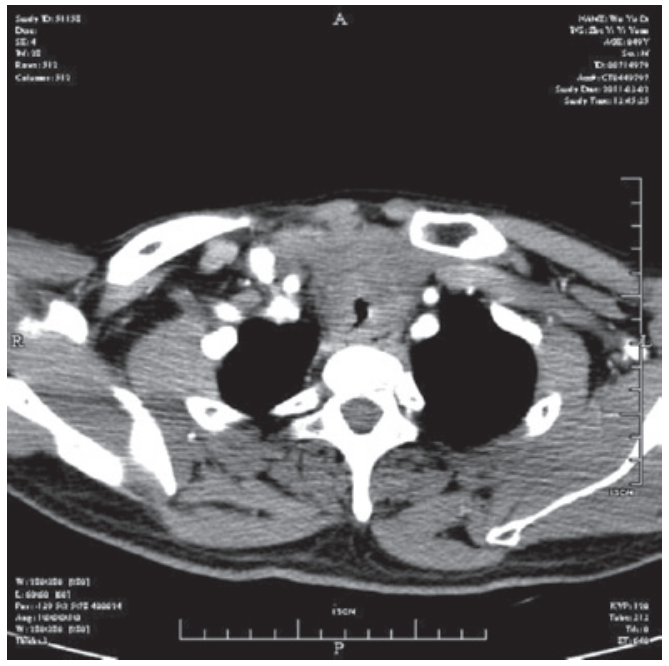

Figure 1. CT scan showing the tumor had compressed and invaded the trachea. The narrowest stenosis was $0.3 \mathrm{~cm}$ in diameter (Mar. 2, 2011). CT, computed tomography.

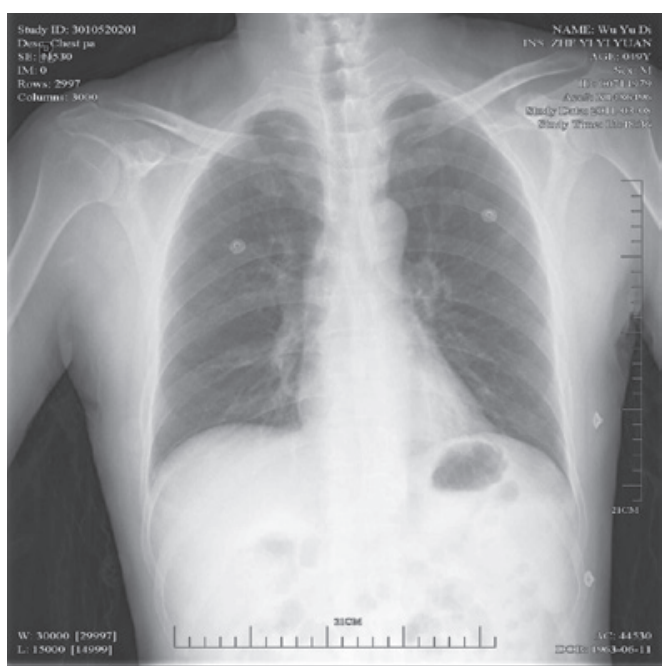

Figure 2. Chest radiograph scan showing the position of the tracheal stent (Mar. 10, 2011).

Management procedure. As the intubation was unable to pass through the narrowest part of the trachea, conventional general anesthesia by tracheal intubation could not be carried out and it was also unsafe, as intubation may have resulted in suffocation and mortality. As the tumor had invaded the trachea and extended to the substernal area, tracheotomy alone may not have been sufficient to relieve the breathing difficulties. Suffocation may also have occurred when undergoing flexible bronchoscopy. Therefore, extracorporeal oxygenation support was thought to be necessary for the patient.

The patient was sedated with intravenous diazepam. Under local anesthesia with lidocaine, a femoro-femoral cardiopulmonary bypass was started; venous drainage was obtained by right femoral vein cut-down and arterial inflow was obtained by right femoral artery cut-down. Through the cardiopulmonary bypass system, the level of arterial oxygen saturation $\left(\mathrm{SaO}_{2}\right)$ was maintained at $>90 \%$ throughout the procedure. The patient was intravenously anesthetized and

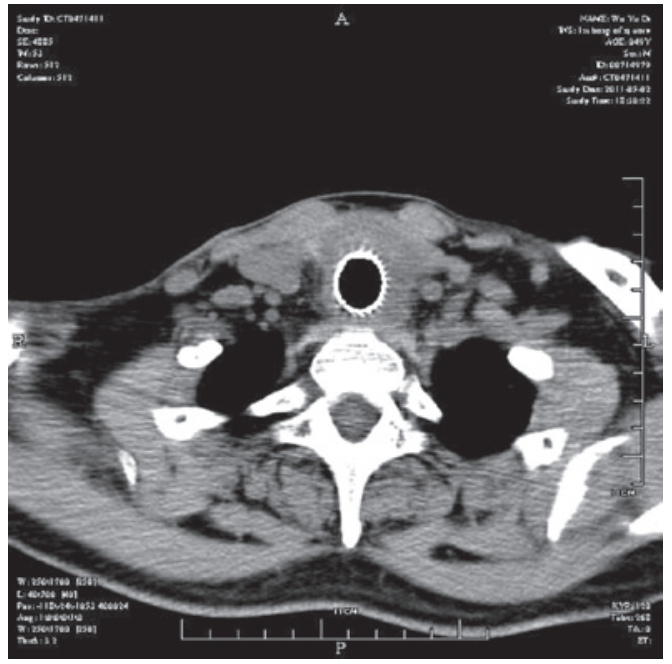

Figure 3. CT scan after two cycles of concurrent chemoradiotherapy showing that the stent was successfully implanted and open to maintain patency of the tracheal lumen (May 2, 2011). CT, computed tomography.

flexible tracheoscopy was performed, which revealed a severe narrowing of the trachea at $5 \mathrm{~cm}$ from the vocal cords with invasion by tumor tissue, and the narrowest stenosis was $0.3 \mathrm{~cm}$ in diameter. Attempts to traverse the stenosis with a bronchoscope were unsuccessful. Incisional biopsy was performed guided by a bronchoscope and tumor tissue was acquired. Fast-frozen pathology revealed poorly differentiated thyroid carcinoma. Therefore, it was decided to insert a tracheal stent to treat the compromised airway instead of performing a surgical tumor resection. A guide-wire was inserted through the fiber optic bronchoscope into the trachea stenosis. Then the bronchoscope was moved away, and an $18 \times 60-\mathrm{mm}$ stent was inserted into the trachea under fluoroscopic control. Finally, the distal end of the stent was located across the stricture to normal trachea tissue. The patient experienced neither dyspnea nor stridor, the respiratory function was significantly improved and there were no complications following stent insertion (e.g., migration, retention of secretions). Furthermore, a chest radiograph scan revealed that the stent was successfully implanted in the trachea (Fig. 2).

Subsequently, the patient was scheduled for three cycles of concurrent chemoradiotherapy and three cycles of chemotherapy alone.

Results. Immunohistochemical analyses revealed that the tumor was a poorly differentiated carcinoma with positive expression of CK14 and P63 and negative expression of TTF-1 (thyroid transcription factor-1) and SP-A (surfactant protein-A). Subsequently, the patient was scheduled for concurrent chemoradiotherapy. After two cycles of concurrent chemoradiotherapy, a cervical CT scan revealed that the size of the tumor was significantly reduced, the stent was open to maintain patency of the lumen and there was no granulation tissue or other abnormal lesions in the tracheal lumen (Fig. 3). Six months after surgery, a CT scan showed that there were no tumor recurrences in the trachea (Fig. 4).

To date, the patient has survived for ten months and finished chemoradiotherapy. A CT scan showed that the thyroid tumor appeared to be more localized and reduced, compared with 


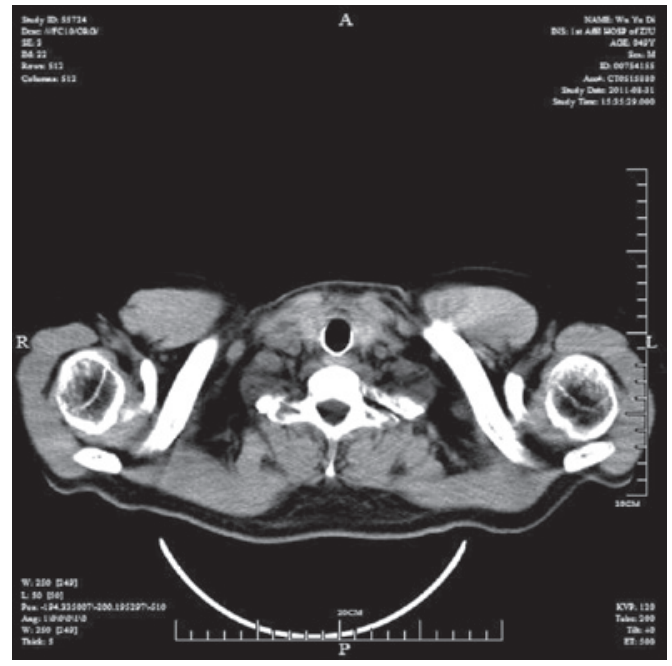

Figure 4. CT images six months after surgery, showing that the airway was open and there was no tumor in the trachea (Aug. 31, 2011). CT, computed tomography.

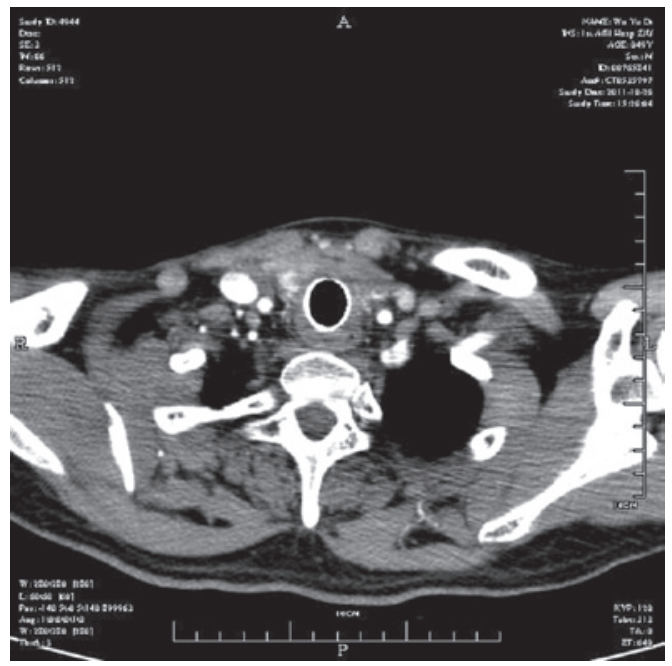

Figure 5. CT scan ten months after surgery, showing that the thyroid tumor appeared to be more localized and reduced than pre-surgery (Oct. 27, 2011). CT, computed tomography.

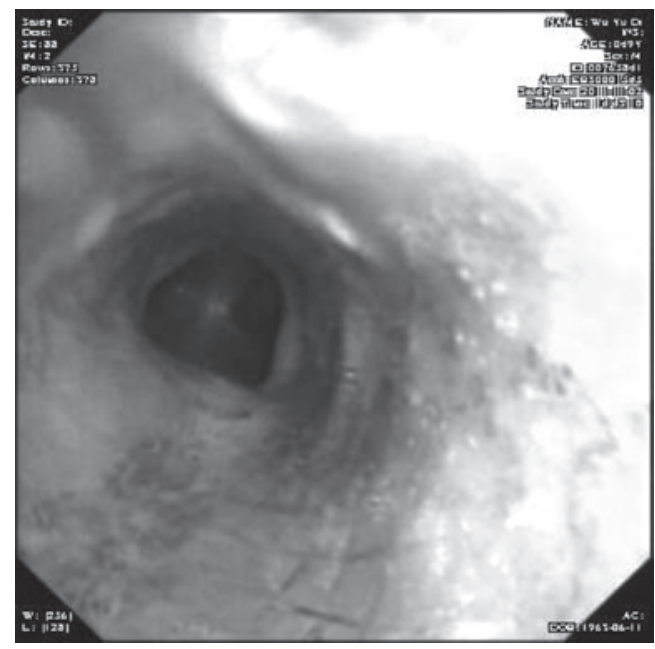

Figure 6. Bronchoscopic examination showed that the airway was smooth around the endobronchial stent (Nov. 2, 2011). the previous CT scan (Fig. 5). Bronchoscopic examination showed that the airway was smooth around the endobronchial stent (Fig. 6).

\section{Discussion}

Poorly differentiated thyroid carcinoma is a group of carcinomas that ranks between well-differentiated and anaplastic thyroid carcinoma in terms of both morphological appearance and biological behavior $(1,2)$. An international panel of 12 thyroid pathologists recommended a diagnostic algorithm ('Turin Proposal criteria') on the basis of the interpretation of the WHO classification and suggested a uniform approach to classify these tumors. The Turin proposal algorithm defines poorly differentiated carcinomas by the presence of a solid/trabecular/insular growth pattern, absence of conventional nuclear features of papillary carcinoma and the presence of at least one of the following features: convoluted nuclei, mitotic activity greater than or equal to $3 / 10 \mathrm{HPF}$ or tumor necrosis (3).

Airway obstruction usually occurs secondary to malignant cervical disease. In the case of an almost completely occluded trachea, endotracheal intubation may be unexpectedly difficult, which may result in catastrophic outcomes, including cerebral anoxia and mortality. In the present study, due to the massive tracheal invasion, the narrowest stenosis was $0.3 \mathrm{~cm}$ in diameter and extended to the substernal area, so tracheotomy alone may have been unable to relieve the breathing difficulties. Suffocation may also have occurred when undergoing flexible bronchoscopy. Therefore, proper evaluation and timely intervention are decisive for such patients. Cardiopulmonary bypass has been applied in cardiac and respiratory failure for a number of years. Venous blood is removed from the body, oxygenated and then returned to the body. In the field of malignant airway obstruction, cardiopulmonary bypass has been used for the management of airway in a few case reports. Sendasgupta et al (4) reported the successful management of a patient with a large anterior mediastinal mass which compressed and distorted the trachea through a femoro-femoral cardiopulmonary procedure. In a patient with an aggressive thyroid papillary carcinoma invading the trachea, curative surgery and reconstruction were performed by cardiopulmonary bypass oxygenation (5). Tyagi et al reported that two patients who presented with respiratory distress with cyanosis had a pedunculated mass in the lower trachea above the carina, with approximately $90 \%$ tracheal lumen obstruction. The two patients were successfully managed with femoro-femoral cardiopulmonary bypass and the airways were restored (5). As described in the present study, cardiopulmonary bypass provides adequate oxygen support to facilitate further appropriate treatment in patients with massive tracheal invasion.

Tracheal resection and reconstruction with primary anastomosis is considered to be the curative treatment for gross intraluminal involvement of malignant tumors. However, the indications are rigid: the glottis must be functional and patients should not have severe pulmonary pathology (6). To avoid circumferential mobilization of the trachea or larynx, a total of 5 to $6 \mathrm{~cm}$ of trahea may be resected and primary reanastomosis performed (7). In the present study, as the 
tumor had invaded a 5-cm stretch of the trachea and extended to the substernal area, tracheal resection and reconstruction was not only difficult but also dangerous. Tracheal stent implantation is an effective palliative treatment for patients with malignant tracheal obstruction, especially for inoperable patients (8-10). It significantly relieves acute dyspnea caused by local tumor compression or invasion and improves the quality of life of patients. The stent may also function as a bridge until further curative treatment may be administered. As expected, this patient's symptoms, including dyspnea and stridor, were significantly relieved through the placing of a self-expanding metallic stent. At present, the application of the non-membrane coated metallic stent is somewhat controversial in malignant airway obstruction. Certain experts consider that, unlike a covered stent, non-membrane coated metallic stents are unable to hinder the overgrowth of granulation tissue, leading to difficulties with intubation or ventilation (11). However, others have reported (8) that tumors do not grow into the non-membrane coated metallic stent as easily as expected, as, unlike the uncoated stent, the covered stent does not compress the tumor. On the contrary, the nonmembrane coated stent results in the avascular necrosis of the tumor.

As the stent implantation keeps the respiratory tract open, this provides time to undergo further management of the tumor $(10,12)$. Adjuvant therapy and/or chemotherapy appear to be effective in patients with poorly differentiated thyroid carcinoma. In particular, endoluminal brachytherapy may reduce the risk of granulation tissue formation above and below the mesh stents and neoplastic infiltration through the mesh into the lumen (13). In the present study, after two cycles of concurrent chemoradiotherapy, the size of the tumor was significantly reduced, the stent was open to maintain the patency of the lumen and there was no granulation tissue or other abnormal lesions in the tracheal lumen.

In conclusion, for patients with serious tracheal obstruction secondary to a malignant tumor, femoro-femoral cardiopulmonary bypass provides adequate oxygen support to facilitate further management, such as tumor resection or chemoradiotherapy, so as to reduce or cure the tumor. Tracheal stent implanting is an effective emergent measure to relieve tracheal obstruction.

\section{Acknowledgements}

We thank Xuefen Wang, Department of Pulmonary Medicine, and Liping Shi, Department of Extracorporeal Crculation, The First Affiliated Hospital, Zhejiang University College of Medicine for providing technological assistance.

\section{References}

1. Patel KN and Shaha AR: Poorly differentiated and anaplastic thyroid cancer. Cancer Control 13: 119-128, 2006.

2. Sanders EM Jr, LiVolsi VA, Brierley J, et al: An evidence-based review of poorly differentiated thyroid cancer. World J Surg 31: 934-945, 2007.

3. Volante M, Collini P, Nikiforov YE, et al: Poorly differentiated thyroid carcinoma: the Turin proposal for the use of uniform diagnostic criteria and an algorithmic diagnostic approach. Am J Surg Pathol 31: 1256-1264, 2007.

4. Sendasgupta C, Sengupta G, Ghosh K, Munshi A and Goswami A: Femoro-femoral cardiopulmonary bypass for the resection of an anterior mediastinal mass. Indian J Anaesth 54: 565-568, 2010.

5. Tyagi I, Goyal A, Syal R, Agarwal SK and Tewari P: Emergency cardiopulmonary bypass for impassable airway. J Laryngol Otol 120: 687-690, 2006.

6. Pinsonneault $\mathrm{C}$, Fortier J and Donati F: Tracheal resection and reconstruction. Can J Anaesth 46: 439-455, 1999.

7. Price DL, Wong RJ and Randolph GW: Invasive thyroid cancer: management of the trachea and esophagus. Otolaryngol Clin North Am 41: 1155-1168, ix-x, 2008.

8. Ma G, Wang DF, Su QG, et al: Tracheal stent implantation for the treatment of tumor-induced acute airway stenosis. Ai Zheng 27: 851-855, 2008 (In Chinese).

9. Mroz RM, Kordecki K, Kozlowski MD, et al: Severe respiratory distress caused by central airway obstruction treated with selfexpandable metallic stents. J Physiol Pharmacol 59 (Suppl 6): 491-497, 2008.

10. Kim JH, Shin JH, Song HY, et al: Palliative treatment of inoperable malignant tracheobronchial obstruction: temporary stenting combined with radiation therapy and/or chemotherapy. AJR Am J Roentgenol 193: W38-42, 2009.

11. Davis N, Madden BP, Sheth A and Crerar-Gilbert AJ: Airway management of patients with tracheobronchial stents. Br J Anaesth 96: 132-135, 2006.

12. Lemaire A, Burfeind WR, Toloza E, et al: Outcomes of tracheobronchial stents in patients with malignant airway disease. Ann Thorac Surg 80: 434-437; discussion 437-438, 2005.

13. Yerushalmi R, Fenig E, Shitrit D, et al: Endobronchial stent for malignant airway obstructions. Isr Med Assoc J 8: 615-617, 2006. 